\title{
Application of Machine Learning Tools for the Improvement of Reactive Extrusion Simulation
}

\author{
Fanny Castéran, Ruben Ibanez, Clara Argerich, Karim Delage, Francisco Chinesta, and \\ Philippe Cassagnau*
}

The purpose of this paper is to combine a classical 1D twin-screw extrusion model with machine learning techniques to obtain accurate predictions of a complex system despite few data. Systems involving reactive polyethylene oligomer dispersed in situ in a polypropylene matrix by reactive twin-screw extrusion are studied for this purpose. The twin-screw extrusion simulation software LUDOVIC is used and machine learning techniques dealing with low data limit are used as a correction of the simulation.

\section{Introduction}

Reactive extrusion is known to be an efficient and economical way of reactive processing of thermoplastic polymers. Corotating and counter-rotating twin-screw extrusion in particular are widely used for bulk polymerization, chemical modification of polymers and reactive compatibilization of polymer blends.

Whereas polymer synthesis and post synthesis modifications were originally exclusively carried out in batch mixers, some valuable features of extrusion process make it an advantageous alternative for some of these reactions. As a matter of fact, aside from being fast and continuous, this process makes possible to perform polymerizations or chemical reactions without any organic solvent (environment-friendly). It is also an efficient way to devolatilization, which can be useful to remove volatile organic compounds (VOC) such as monomers or reaction byproducts. Moreover, the adaptability of the screw design helps the formulation of complex materials. The screw profile can indeed be specifically designed for each formulation and define different areas of feeding, kneading, shearing, or conveying, depending on the expected final properties of the material. ${ }^{[1]}$

As reported in the literature, all these features have been widely exploited through a large number of chemical reactions and polymer systems studied. ${ }^{[2-6]}$ However, some disadvantages of using the extruder as a reactor must be considered: While

F. Castéran, K. Delage, Prof P. Cassagnau

Univ-Lyon, Université Claude Bernard Lyon 1

Ingénierie des Matériaux Polymères, CNRS

UMR 5223, 15 Bd Latarjet, Villeurbanne Cedex 69622, France

E-mail: philippe.cassagnau@univ-lyon1.fr

Dr. R. Ibanez, Dr. C. Argerich, Prof. F. Chinesta

PIMM Lab, Arts et Métiers Institute of Technology

151 Boulevard de l'Hôpital, Paris 75013, France the extrusion process is well suited for the processing of highly viscous materials, the high shear can still cause self-heating of the material and possible side reactions. In addition, the short residence time limits the number of possible reactions, and finally, the scale up to industrial pilot and plants comes with many difficulties.

The nature of the reactive extrusion process involves a complex flow and a large number of parameters, dependent variables and phenomena that make it difficult to understand and therefore to control and to optimize. In response to this complexity, various strategies have been adopted for the modeling and simulation of reactive extrusion. ${ }^{[5]}$ Two main methodologies can be identified.

The first strategy is based on a chemical engineering approach. This method consists in considering the extruder as a succession of ideal chemical reactors, which number and nature depend on the screw profile geometry. A global balance can then lead to an approximation of flow conditions. This method has been used in several studies, and among them, Choulak et al. used it to develop a dynamic 1D model for automatic control of reactive extrusion. [7] This approach is especially well adapted for automatic control because of its fast execution due to the simplifications and its ability to perform in transient regime. However, it requires to adjust parameters to each situation so it cannot be easily used as a predictive tool or to solve scale up issues.

The second strategy is a local description of the flow field based on continuum mechanics. It thus reproduces real conditions without ideal representation. The flow is indeed simulated by resolving classical mechanics equations relatively to local geometry, kinematics, and boundary conditions. These models can therefore be totally predictive and can be used for process design, but also for its optimization and predictions in case of a change in the process. Whereas it is a more flexible and accurate way to simulate the extrusion process, it requires a lot of time and computing power and is consequently not adapted for automatic process control.

As the flow in the extruder is unsteady, not isothermal, and $3 \mathrm{D}$, it is more accurate to use a $3 \mathrm{D}$ local simulation. But in some cases it appears that a 1D local description of the flow and temperature field at steady-state can be sufficient for most engineering issues. ${ }^{[8]}$ In addition, it allows creating a software easily usable for process predictions without needing excessive time or computing power. We used for our simulation the LUDOVIC software developed by Vergnes et al. for twinscrew extrusion that uses this 1D local description. ${ }^{[9]}$ Actually, 
because of the additional complexity of reactive extrusion, most simulations of reactive extrusion are based on simplified steady state 1D models. It however involves coupling with several submodels to obtain constitutive equations (viscosity, chemical kinetics, mass and temperature transfers...), which is not trivial and takes time. ${ }^{[10]}$ Yet, despite many researches and published papers, there is a lack of describing complex polymer formulations such as reactive extrusion systems.

In these circumstances and given the progress made today in artificial intelligence tools dealing with small amounts of data, it seems interesting to try developing models based on data to reinforce possible weaknesses in existing physical models, to enrich the models when we are confident in the data collected, or even to improve the data when they present a large variability. Finally, the aim of this work is to combine the logic of physical models with the accuracy of machine learning techniques to describe the reactive extrusion of a complex system. This reactive system is based on the in situ reaction of a reactive polyethylene oligomer with a triamine or a sorbitol while dispersing in a polypropylene (PP) matrix.

\section{Experimental Section}

In this work, reactive systems that are model systems, but also complex were chosen to study, in order to justify the use of artificial intelligence methods. The chosen systems consist of dispersing a polymer network resulting in the in situ reaction of a polyethylene oligomer grafted with maleic anhydride and a triamine or a sorbitol in a PP matrix. The reactions were performed by a one-step reactive extrusion process. Several process parameters were tested in order to analyze their influence on the final materials. The processes were also simulated thanks to the software LUDOVIC, whose results have then been compared with the experiments. Finally, machine learning techniques were employed in order to improve the simulation by bringing a correction to its outputs.

\subsection{Materials}

In the present study, a system previously developed in lab in the framework of Ph.D. thesis was used. ${ }^{[11]}$ Actually, the reaction between a linear polyethylene oligomer grafted with maleic anhydride derivates (Ceramer 1608, Baker Hughes) and a trifuctional polyetheramine oligomer (Jeffamine T-403, Huntsman) was studied. The reaction results in a crosslinked network with an equilibrium storage modulus value similar to synthetic rubbers $\left(\mathrm{Ge} \approx 2 \times 10^{6} \mathrm{~Pa}\right.$ ). The objective of the present paper was to perform in situ this reaction in a PP matrix by reactive extrusion. A similar system was also studied by replacing the triamine with a Sorbitol (D(-)-Sorbitol, VWR Chemicals).

The PP used in this work was an isotactic homopolymer (PPH7060, Total). It has a melting temperature of $165^{\circ} \mathrm{C}$ and a density of $0.905 \mathrm{~g} \mathrm{~cm}^{-3}$. Its melt flow index was $12 \mathrm{~g} / 10 \mathrm{~min}$ $\left(230^{\circ} \mathrm{C}, 2.16 \mathrm{~kg}\right)$, which corresponds to a zero shear viscosity of $3.10^{3} \mathrm{~Pa} \mathrm{~s}\left(\mathrm{~T}=180^{\circ} \mathrm{C}\right)$.

The Ceramer 1608 from Baker Hughes used in this study was a solid at room temperature. It has a melting point of $75^{\circ} \mathrm{C}$
Table 1. Chemical structures of the reactants constituting the reactive systems.

Name
Ceramer
$\left(\begin{array}{c}\mathrm{m}+n+p=50, \\ \mathrm{R}=\mathrm{H} \text { or } \mathrm{Pr})\end{array}\right.$

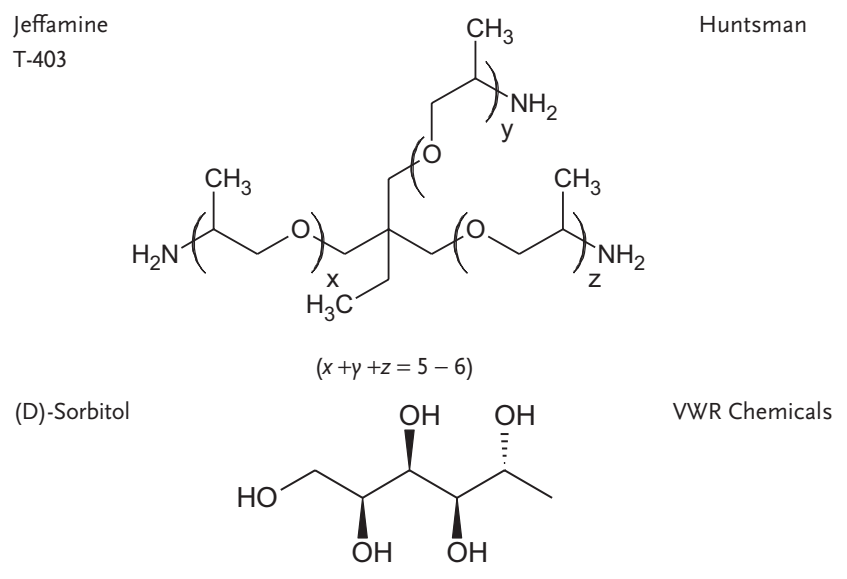

and a zero shear viscosity of $\eta_{0}=2 \mathrm{~Pa} \mathrm{~s}\left(T=180^{\circ} \mathrm{C}\right)$. The technical datasheet provided by the supplier specified an acid value of $154 \mathrm{mg} \mathrm{KOH} / \mathrm{g}$ and a saponification value of $215 \mathrm{mg} \mathrm{KOH} / \mathrm{g}$. The datasheet also specified that the maleic anhydride derivates used for the functionalization of Ceramer 1608 were not only maleic anhydride but also mono-isopropyl maleate ( $\mathrm{Z}$ and $\mathrm{E}$ isomers) and maleic acid ( $\mathrm{Z}$ and $\mathrm{E}$ isomers). For simplification purposes, maleic anhydride will be considered as the only maleic anhydride derivate of this functionalized oligomer.

The Jeffamine T-403 trifunctional polyetheramine oligomer from Huntsman was liquid at room temperature. It has a density of $0.978 \mathrm{~g} \mathrm{~cm}^{-3}$ with a zero shear viscosity of $\eta_{0}=0.07 \mathrm{~Pa} \mathrm{~s}\left(25^{\circ} \mathrm{C}\right)$, a molar mass of $400 \mathrm{~g} \mathrm{~mol}^{-1}$, and a amine hydrogen equivalent weight of $81 \mathrm{~g} \mathrm{eq}^{-1}$ according to its technical datasheet.

The Sorbitol was conditioned as a white solid powder. The chemical structures of the reactants are presented in Table 1.

The amount of functional groups in the oligomers has been determined from the acid and saponification values in the case of the Ceramer 1608 and from the amine hydrogen equivalent weight in the case of Jeffamine T-403. In the case of Sorbitol, only the two primary alcohol functions were considered.

The reagent ratios of the two systems were determined assuming near stoichiometry. The ratio in weight of Ceramer 1608 against Jeffamine T-403 was set at 75/25, and the one of Ceramer 1608 against Sorbitol was set at 85/15.

The reaction mechanisms are shown in Figure 1 . The reaction between the Ceramer and the triamine involved a reaction between the maleic anhydride groups of the Ceramer and the amine groups of the Jeffamine, yielding a cyclic imide moiety along with water as a by-product. As for as the reaction between the Ceramer and the Sorbitol, the maleic anhydride cycle was opened, forming an ester function and a carboxylic acid function. 

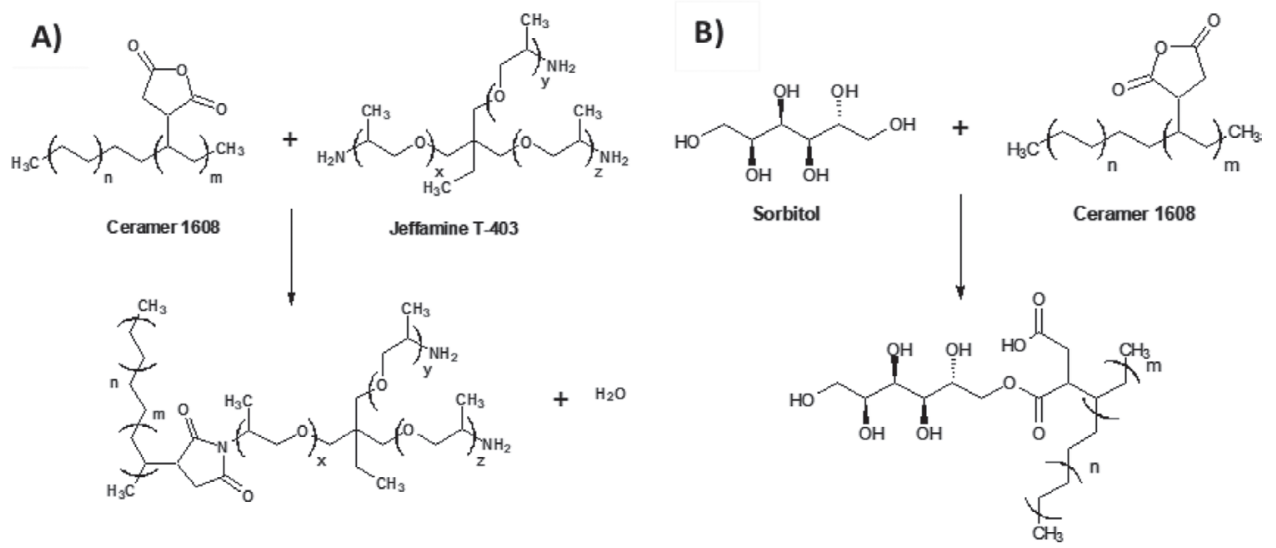

Figure 1. A) Reaction between Ceramer 1608 and Jeffamine T-403 oligomers; B) Reaction between Ceramer 1608 and Sorbitol.

These reactive systems were quite complex due to their chemistry and the solubility of the reagents, and it was therefore difficult to transcribe the real conditions in a simulation such as LUDOVIC in terms of, for example, network formation (reaction conversion) as a function of time and temperature. This was where data science and machine learning become interesting to use to improve existing simulations.

\subsection{Process}

The chemical extrusion was carried out in one single step with a co-rotating twin-screw extruder (Leistritz ZSE18, L/D = $60, D=18 \mathrm{~mm}$ ). The PP pellets and Ceramer were introduced together at the entrance by a hopper, the Sorbitol was introduced at the same spot with a powder feeder, and the Jeffamine was added once passed the melting zone at bloc 3 by means of a HPLC pump. A vent was placed over the second last bloc in order to remove the gases generated during the process. The final materials were cooled by air at the die exit of the extruder and then pelleted. All the experiments were carried out at $200{ }^{\circ} \mathrm{C}$ with the first blocs at lower temperature to minimize clogging effects at the inlet. The temperature and screw profiles used for all the experiments are described in Figure 2.

The formulations with the system Ceramer/Jeffamine dispersed in PP were carried out as specified in Table 2, with screw rotation speeds from $N=200$ to $1000 \mathrm{rpm}$ and exit flow rate from $\dot{w}=3$ to $20 \mathrm{~kg} \mathrm{~h}^{-1}$. Two formulations were tested for this system with $\phi=15 \mathrm{wt} \%$ and $\phi=30 \mathrm{wt} \%$ of dispersed phase. Concerning the system Ceramer/Sorbitol, it was dispersed at $\phi=20 \mathrm{wt} \%$ in PP for different processing conditions: screw rotation speeds of $N=300$ and $800 \mathrm{rpm}$, and exit flow rates of $\dot{w}=5 \mathrm{~kg} \mathrm{~h}^{-1}$ and $\dot{w}=10 \mathrm{~kg} \mathrm{~h}^{-1}$.

Temperature and pressure were measured at the die entrance. However, the thermocouple used inside the die did not measure the true temperature at the core of the material but the temperature on the sides, thus influenced by the die temperature. Some temperatures were then measured at the exit by means of a manual thermocouple. The experiments performed are summarized in Table 2.

\subsection{Characterizations}

Tensile-test pieces (5A) were injected with a Babyplast injection press at $200{ }^{\circ} \mathrm{C}$ and 90 bar. Young modulus was determined

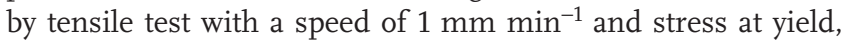
elongation at break, and stress at break were measured with a tensile speed of $50 \mathrm{~mm} \mathrm{~min}^{-1}$.

Morphologies were characterized by scanning electronic microscopy (SEM) with ZEISS MERLIN COMPACT VP. The samples were prepared from the injected tensile-test pieces

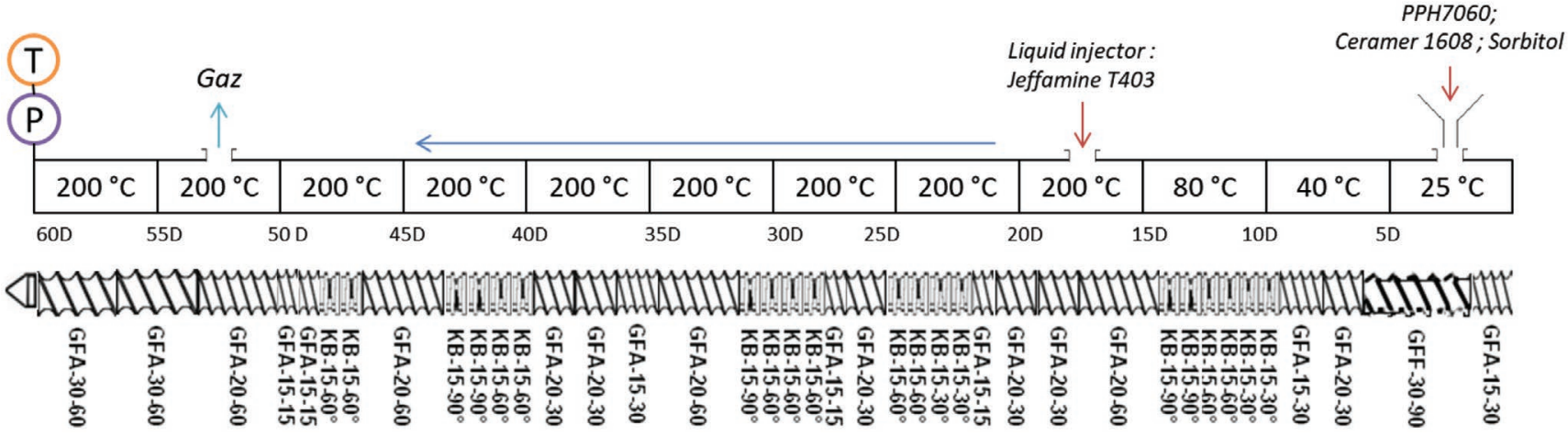

Figure 2. Scheme of the screw configuration used in the study. 
Table 2. Formulations and processing conditions for the different reactive blends.

\begin{tabular}{|c|c|c|c|}
\hline System studied & $\%$ Dispersed phase $\phi$ & Exit flow rate $\dot{w}\left[\mathrm{~kg} \mathrm{~h}^{-1}\right]$ & Rotation speed $N$ [rpm] \\
\hline $75 / 25$ & $15 \%$ & 3 & 200 to 1000 \\
\hline \multirow{2}{*}{$\begin{array}{l}\text { Ceramer } 1608 \\
\text { Jeffamine T-403 } \\
+\end{array}$} & \multirow{5}{*}{$\begin{array}{l}\text { (12\% Ceramer } 1608 ; \\
3 \% \text { Jeffamine T-403) }\end{array}$} & 5 & 200 to 1000 \\
\hline & & 8 & 300 to 1000 \\
\hline \multirow[t]{7}{*}{ PPH7060 } & & 10 & 600 and 1000 \\
\hline & & 15 & 600 and 1000 \\
\hline & & 20 & 1000 \\
\hline & \multirow{4}{*}{$\begin{array}{c}30 \% \\
(23 \% \text { Ceramer } 1608 ; 7 \% \text { Jeffamine T-403) }\end{array}$} & 3 & 150 to 600 \\
\hline & & 5 & 600 and 1000 \\
\hline & & 8 & 600 and 1000 \\
\hline & & 10 & 600 and 1000 \\
\hline $85 / 15$ & $20 \%$ & 5 & 300 and 800 \\
\hline Ceramer 1608 & (17\% Ceramer 1608; & 10 & 300 and 800 \\
\hline Sorbitol & 3\% Sorbitol) & & \\
\hline \multicolumn{4}{|l|}{+} \\
\hline PPH7060 & & & \\
\hline
\end{tabular}

(5A). They were then fractured in liquid nitrogen and metallized with $10 \mu \mathrm{m}$ of a copper coating.

The kinetics of the chemical reaction leading to the formation of a chemical network was studied from rheological studies using an Ares-G2 strain controller rheometer using a geometry of parallel plates of $25 \mathrm{~mm}$ diameter and a gap of $1 \mathrm{~mm}$ spacing. The variation over time of the rheological behavior of the premixed reactants were thus measured, under an angular frequency of $1 \mathrm{rad} \mathrm{s}^{-1}$ at 140 and $180{ }^{\circ} \mathrm{C}$ under nitrogen. The Ceramer and the Jeffamine were premixed in an internal mixer at $T=110{ }^{\circ} \mathrm{C}$ and $50 \mathrm{rpm}$ for $2 \mathrm{~min}$. The Ceramer and the Sorbitol were first premixed at $T=140{ }^{\circ} \mathrm{C}, N=300 \mathrm{rpm}$, and for a $\dot{w}=3 \mathrm{~kg} \mathrm{~h}^{-1}$ flow rate in the extruder previously described. They were then mixed in an internal mixer at $T=140{ }^{\circ} \mathrm{C}$ and $N=60 \mathrm{rpm}$ for $5 \mathrm{~min}$ to extract the gases that formed bubbles inside the material and may alter the measures.

The viscoelastic behavior of the materials was studied with frequency sweep measurements using plate geometry of $8 \mathrm{~mm}$ diameter and $1 \mathrm{~mm}$ gap. A frequency sweep was applied from 100 to $0.01 \mathrm{rad} \mathrm{s}^{-1}$ under a strain of $2 \%$ (linear regime). These tests were performed on the resulting samples of the kinetics measurements.

\section{Modeling and Machine Learning}

\subsection{Simulation}

The LUDOVIC simulation software that we used here has been developed to simulate the flow along a screw profile corresponding to industrial reality. It was also important to be executable on a personal computer without needing excessive computation time or power. To meet these expectations, LUDOVIC uses a global model describing the total flow by linking local 1D models. Various singular strategies and simplifications have therefore been used to achieve sufficient precision despite the 1D considerations described below.
It is commonly admitted in these process conditions that the material melting occurs rapidly after reaching the first restrictive element. Consequently, this phenomenon is by default assumed instantaneous in the simulation. Meanwhile, it is possible for the user to import a specific melting model.

Concerning then the melt conveying section, two main different elements have to be considered and require distinct methodologies: the screw elements and the kneading discs (left and right-handed). The screw elements are often treated in 1D or 2D using a model flattening out the screw channel with a sliding barrel moving at the top. Yet, the strategy employed in this software uses a different 1D model following cylindrical coordinates along an eight-shaped pattern representing the screw channel. The channel section is considered to be rectangular and perpendicular to the screw flights with a constant width and Stokes equations are solved in this section. The kneading discs are also usually simulated using 2D approaches but a $1 \mathrm{D}$ approach is necessary to ease the calculations. Based on previous studies, only the peripheral flow in the $\theta$-direction around a disc is thus considered. The representations of both these elements are schematized in Figure 3.

In these configurations, the flow is locally calculated as Newtonian and isothermal. It is however expressed following Newtonian flow equations that include a specific viscosity that is a function of the shear rate and the temperature. This viscosity can be then defined apart and follow specific rheological models such as power law, Carreau-Yasuda or Casson. The mean viscosity in channel direction and the mean temperature can be then calculated. A 1D approach is used to define the temperature. As the screws in a twin-screw extruder are not totally filled, the filling ratio is unknown. Consequently, the calculation has to follow an iterative procedure: The die temperature is randomly defined, and the simulation is run backward the flow, from the die until the feeding section. If the final pressure and temperature do not match the expected inlet ones, the exit temperature is adjusted, and the calculations are reiterated until the match with the inlet conditions. The exact equations 

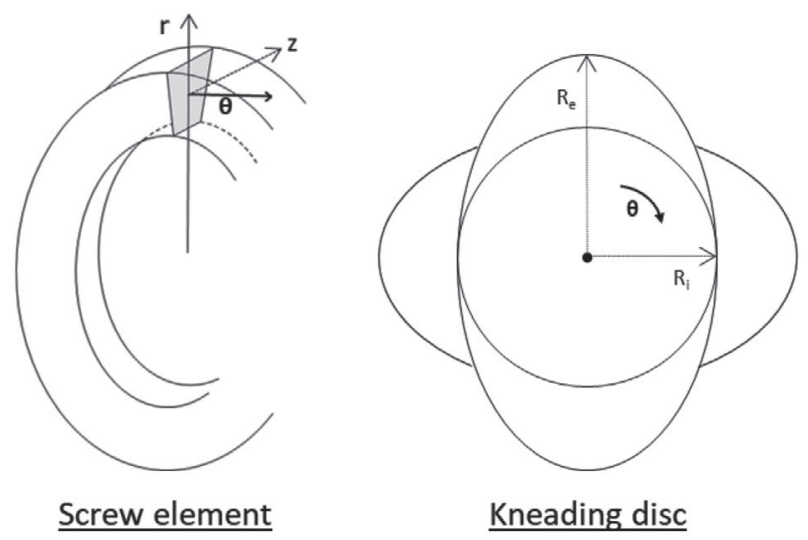

Figure 3. Geometries of a screw element (the C-shaped chamber) and a bilobal kneading disc. ${ }^{[9]}$

and methods used in the simulation have been fully detailed by Vergnes et al. in their article. ${ }^{[9]}$

Despite all these drastic simplifications, LUDOVIC software has been validated by numerous experiments and compared with 3D simulations ${ }^{[12,8]}$ It has also been widely used since its creation in various extrusion domains, such as polymer blends, in situ nanocomposites, extrusion cooking of cereal-based products, and more recently, glass and plant fibers break during compounding. ${ }^{[13-17]}$

For this study, the screw profile, extruder and die dimensions as well as barrel temperature were defined to correspond to our experiments. With respect to the material properties, since the software does not consider a dispersed phase, we assumed that the rheological behavior of the formulation is only determined by the matrix. As PP is the matrix, the simulation has been run considering one unique PP phase. The physical properties of this phase were defined from the experiments of Fel et al. who obtained good results using LUDOVIC to simulate twin-screw extrusion of the same PP as the one we used. ${ }^{[18]}$ A CarreauYasuda law was thus applied using the following equation:

$\eta=\eta_{0}(T)\left((1+\lambda(T) \dot{\gamma})^{a}\right)^{\frac{m-1}{a}}$

where $\eta_{0}$ is the zero shear viscosity, $\lambda$ is the relaxation time, $\dot{\gamma}$ is the shear rate, $a$ and $m$ are specific parameters. The values of these parameters and the thermal properties are summarized in Table 3

Finally, the heat transfer coefficient between the polymer and the die have been fixed at $50 \mathrm{~W} \mathrm{~m}^{-2} \mathrm{~K}^{-1}$ and the one between the polymer and the barrel was of $350 \mathrm{~W} \mathrm{~m}^{-2} \mathrm{~K}^{-1}$, which corresponds to values used in similar simulations..$^{[9,19]}$

\subsection{Machine Learning Techniques}

In the past, science was based on the extraction of models, these being simply the causal relation linking causes (inputs) and responses (outputs). This (intelligent) extraction or discovery was performed by smart (and trained) human minds from the data provided by the direct observation of the reality
Table 3. PP thermal properties and Carreau-Yasuda parameters used in the simulation.

\begin{tabular}{|c|c|c|c|c|}
\hline \multirow{2}{*}{$\begin{array}{l}\text { Thermal properties } \\
\left.\text { Heat capacity [J kg-1 } \mathrm{K}^{-1}\right]\end{array}$} & \multirow{2}{*}{$\begin{array}{c}\text { Solid state } \\
2500\end{array}$} & \multirow{2}{*}{$\begin{array}{l}\text { Liquid state } \\
2500\end{array}$} & \multicolumn{2}{|c|}{$\begin{array}{l}\text { Carreau-Yasuda } \\
\text { parameters }\end{array}$} \\
\hline & & & $\eta_{0}[\mathrm{~Pa} \mathrm{~s}]$ & 1000 \\
\hline Density $\left[\mathrm{kg} \mathrm{m}^{-3}\right]$ & 900 & 750 & $\lambda[\mathrm{s}]$ & 0.4 \\
\hline $\begin{array}{l}\text { Thermal conductivity } \\
{\left[\mathrm{W} \mathrm{mK}^{-1}\right]}\end{array}$ & 0.33 & 0.33 & $a$ & 0.21 \\
\hline Melting temperature $\left[{ }^{\circ} \mathrm{C}\right]$ & 165 & & $m$ & 0.6 \\
\hline \multirow[t]{2}{*}{ Melting enthalpy $\left[\mathrm{kJ} \mathrm{kg}^{-1}\right]$} & & 209 & $T_{\text {ref }}\left[{ }^{\circ} \mathrm{C}\right]$ & 200 \\
\hline & & & $\mathrm{Ea}\left[/ \mathrm{mol}^{-1}\right]$ & 40000 \\
\hline
\end{tabular}

or from engineered experimental tests. Then, with the discovered, derived, or postulated model, predictions were performed, leading to the validation or rejection of these models.

Thus, physics-based models, often in the form of partial differential equations, were manipulated by using numerical techniques, with the help of powerful computers. However, sometimes models are not available, or they are not accurate enough. In that case, the most natural route consists of extracting the model from the available data (a number of inputs and their associated outputs). When data is abundant and the time of response is not a constraint, deep-learning could constitute the best alternative. However, some industrial applications are subjected to: i) scarce data and ii) necessity of learning on-the-fly under stringent real-time constraints. There is no free lunch: to compensate the lack of data some extra-knowledge must be incorporated during the model extraction (discovery).

In what follows, we will consider that data and perform efficient regressions for correlating the chosen inputs with the selected outputs. To account the just mentioned difficulty associated with the necessity of proceeding in the low-data limit, we will consider two techniques that the authors proposed recently: i) a sparse collocated parametric separated representation, called SPGD, and ii) a regression based on the construction of goal-oriented non-Euclidean metrics able to compare data, that is calculating the distance between data. The last technique was called Code2Vect. ${ }^{[20,21]}$ Both techniques are revisited and summarized as follows.

\subsubsection{Sparse Regression -sPGD}

Linear regression is the simplest way of linearly relating input parameters with output quantities of interest. For illustrating the procedure (for additional details the interested reader can refer to Ibanez et al. (2018)) we consider a given output (u) expected depending on the two input parameters $p$ and $q$, that is, $u(p, q)$. The simplest regression, as previously indicated consists in the linear relation presented in Equation (2).

$u(p, q)=a+b \times p+c \times q$

If three data is available, that is, the output $u_{1}$ related to the inputs $p_{1}$ and $q_{1}$; the output $u_{2}$ related to $p_{2}$ and $q_{2}$, and finally $u_{3}$ associated with $p_{3}$ and $q_{3}$, one could calculate the three coefficients $(a, b$, and $c)$ in Equation (2). 
However, very probably the parametric dependence $u(p, q)$ is nonlinear, and with no more data, calculating richer regressions seems out of reach. Here a solution exists, the sPGD instead of considering the simple approximation given by (2), considers

$U(p, q)=P(p) \times Q(p)$

that being both functions $P(p)$ and $Q(q)$ unknown defines a nonlinear problem, to be calculated in the solution procedure. In that case one proceeds by linearizing, that is, by assuming $Q(q)$ given and looks for $P(p)$. Thus, for computing $P(p)$ the three available data enables an approximation richer that the linear previously considered. Then, from the just computed $P(p)$ one looks to update $Q(q)$ again with three available data.

Thus, $P(p)$ and $Q(q)$ could be approximated using a quadratic approximation, leading to richer nonlinear approximations. The solution compactness is enforced by maximizing sparsity.

As soon as functions $P(p)$ and $Q(q)$ are calculated, the output $U$ in Equation (3) can be easily and quickly calculated for any value of the inputs $p$ and $q$.

\subsubsection{Code2Vect}

Classification and regression could be facilitated if the outputs difference, for example, $\left|u_{1}-u_{2}\right|$, scales with the data distance, for example, $\left\|p_{1}-p_{2}, q_{1}-q_{2}\right\|$. If the difference of the output scales with the distance between the data-points, as soon as a new data comes, its associated output could be calculated by interpolating the ones at the neighbor data-points.

However, sometimes two data points can be very close while the associated outputs differ considerably. In that case, in Argerich et al. (2019) it was proposed mapping the data point into a reduced space, for example, $\left(p_{i}, q_{i}\right)$ into $z_{i}$, such that the distance $\left|z_{i}-z_{j}\right|$ scales with $\left|u_{i}-u_{j}\right|{ }^{[21]}$ Thus, classification and regression become almost trivial in the $\mathrm{z}$-space. The mapping that transform $(p, q)$ into $z$ can be viewed as the construction of a non-Euclidian metric, and was detailed in Argerich et al. (2019).

\subsubsection{Model-Data Hybridization}

Sometimes one measures data, for example, $u^{\exp }(p, q)$, that differs from a model prediction based on existing knowledge or physics, that provides $u^{\bmod }(p, q)$. When both differ significantly the model prediction cannot be employed anymore without incurring risks. Two valuable gateways consist in:

- Elaborating more accurate models. However, this route implies time and effort.

- Elaborate a model of the measured data, that is a regression for expressing the dependence between $u^{\exp }$ and the parameters, in our example, $p$ and $q$. This pragmatic alternative is confronted to the necessity of having enough data for constructing the regression by using for example one of the techniques previously described (sPGD and Code2Vect).

An appealing alternative route consists in assuming that the measures are expressed from the model and its intrinsic deviation, that is, $u^{\exp }(p, q)=u^{\bmod }(p, q)+C(p, q)$, where $\mathrm{C}$ refers to the correction. By assuming that if the model is a reasonably good approximation of the real system, the correction is much less rich that the data itself, the construction of $C(p, q)$ will require much less data for the same degree of accuracy. Thus, the available data, even if not so big, suffices for creating the regression as discussed before. This is the rationale behind the co-called Hybrid-Twin paradigm, as described in ref. [22].

\section{Results and Discussion}

\subsection{Kinetics Study}

A study of the reactions kinetics of the two reactive systems has been performed according to the rheological method described in the experimental part. As shown in Figure 4, the reaction between Ceramer and Jeffamine is clearly faster than the reaction with the Sorbitol at any temperature, even if the Ceramer has been premixed longer with the Sorbitol than with the Jeffamine.

The final shear storage moduli of these systems are both above $3.10^{6} \mathrm{~Pa}$, with higher values for the Ceramer/Jeffamine system. The viscoelasticity studies are present in Figure 5, they reveal that these reactive systems behave like solids at these temperatures $\left(G^{\prime} \gg G^{\prime \prime}, \tan (\delta) \approx 10^{-2}\right)$ so that we can assume that they form a high-density crosslinked network.

With such systems with a fast kinetics, it is difficult to obtain a relevant kinetic model, and the mixing conditions in the extruder are not well known for these systems. At higher temperatures such as our processing conditions, the reactions are
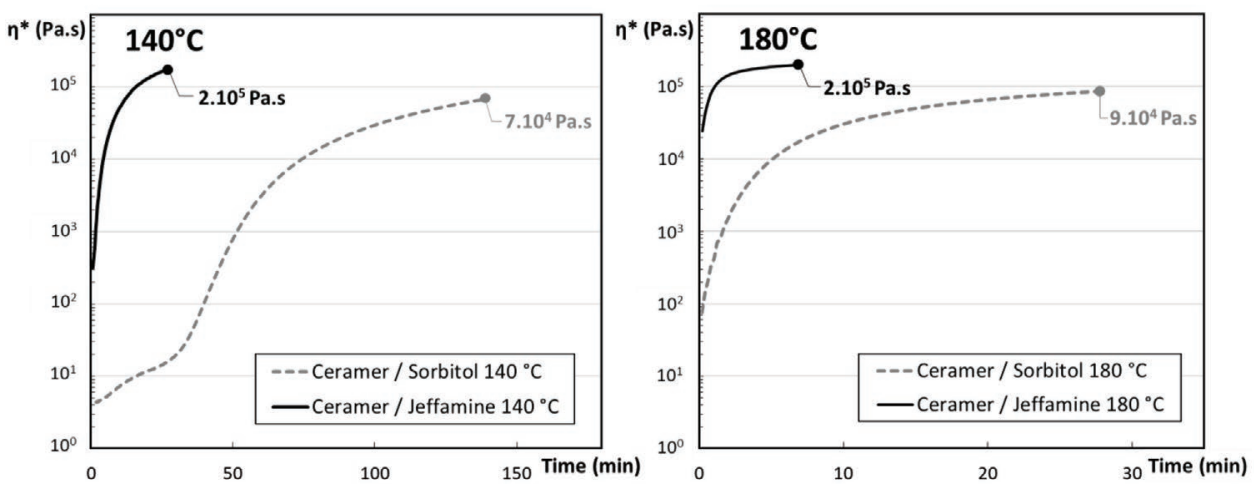

Figure 4. Variation of the absolute complex viscosity versus time for the two reactive systems at the temperature $T=140$ and $180{ }^{\circ} \mathrm{C} ; \omega=1 \mathrm{rad} \mathrm{s}$. 

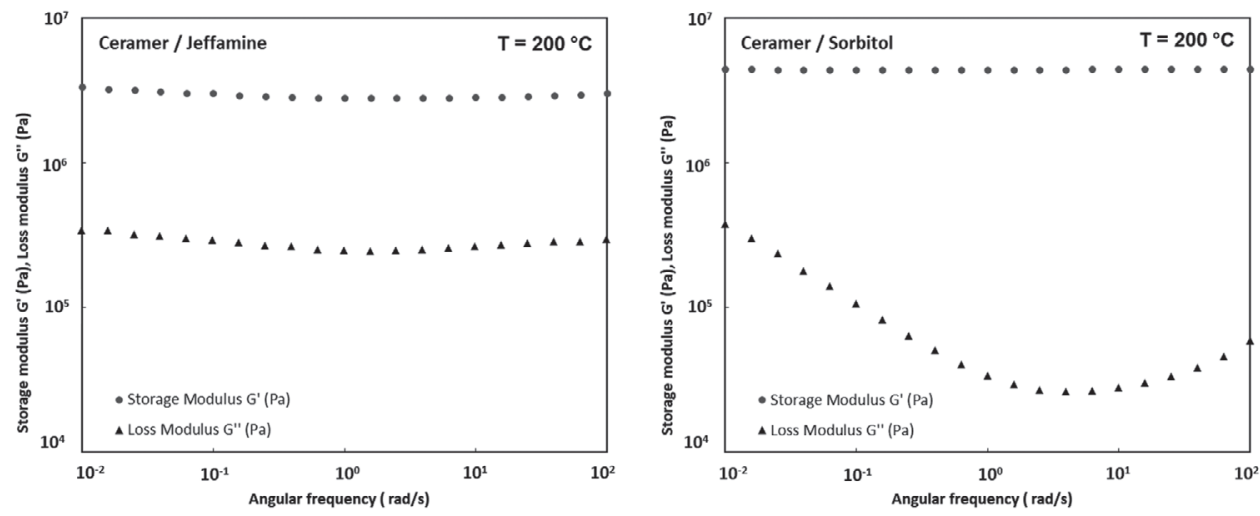

Figure 5. Variation of storage modulus $G^{\prime}$ and loss modulus $G^{\prime \prime}$ versus frequency of the two systems at $T=200^{\circ} \mathrm{C}$.

even faster, and for the Ceramer/Jeffamine system, the reaction occurs before the dispersion in the matrix. The viscosity ratio between the reactive system and the PP matrix is then advantageous for a dispersion in the PP, but as showed later, this results in a coarse morphology. However, the Ceramer/Sorbitol has a slower kinetics that allows a lubrication phenomenon leading paradoxically to a better morphology.

\subsection{Morphologies}

The high reactivity of the dispersed Ceramer/Jeffamine system can be an advantage in reactive extrusion process because of the short residence times (between 15 and $90 \mathrm{~s}$ in the present study), however it can also affect the quality of the dispersion. Actually, the SEM analysis reveals large and deformed nodules (about $10 \mu \mathrm{m}$ ). This phenomenon can be explained by a fast reaction occurring before the system reaches a good dispersion in the matrix. Consequently, solid networks are created and avoid better dispersion afterward.

No real difference in the morphologies can be clearly noticed with the variations of process conditions. The SEM analysis also pointed out a lack of affinity between the matrix and the nodules as we could observe their detachment from the matrix. This lack of affinity can also be a notable factor explaining the shape of the nodules.

The Figure 6 presents images obtained with the samples that have been processed at $\dot{w}=10 \mathrm{~kg} \mathrm{~h}^{-1}$ and $N=600 \mathrm{rpm}$ for $15 \%$ and $30 \%$ of dispersed phase.

The SEM observation performed on the Ceramer/Sorbitol system highlight the same lack of affinity between the different phases. However as expected, the nodules obtained are more spherical with a better dispersion with a characteristic size of $1 \mu \mathrm{m}$. No significant differences in morphologies can be linked to process variations. Therefore, the slower reaction seems to have improved the shape and size of the nodules. But it is worth noting that the dispersion of nodule sizes is quite large, maybe due to not optimized process conditions (screw profile with insufficient shear rate for instance). Figure 7 presents images obtained with the samples that have been processed at $\dot{w}=10$ and $5 \mathrm{~kg} \mathrm{~h}^{-1}$ with $N=300 \mathrm{rpm}$.

\subsection{Mechanical Tests}

To measure the impact of the oligomer network dispersion in the PP matrix, tensile tests have been performed on samples differing
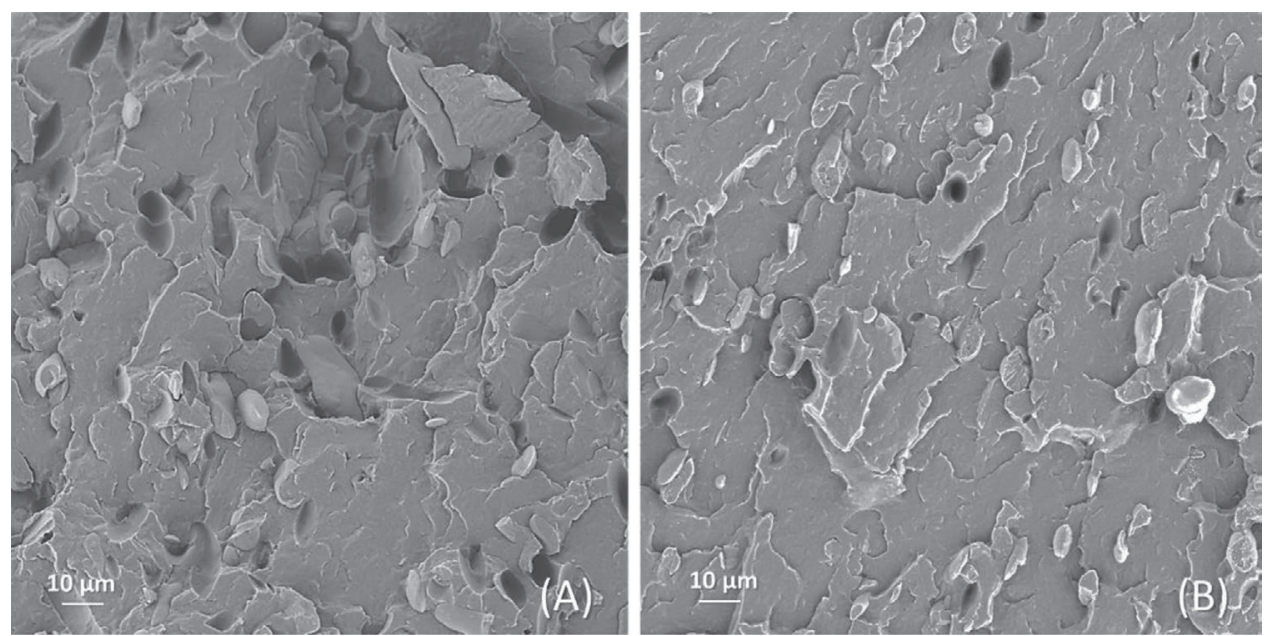

Figure 6. SEM images obtained from the observation of Ceramer/Jeffamine system dispersed in PP; processed at $\dot{w}=10 \mathrm{~kg} \mathrm{~h}{ }^{-1}$ and $\mathrm{N}=600 \mathrm{rpm}$ for A) $30 \%$ and B) $15 \%$ of dispersed phase. Magnitude: $x 500$; Power: 5 kV. 

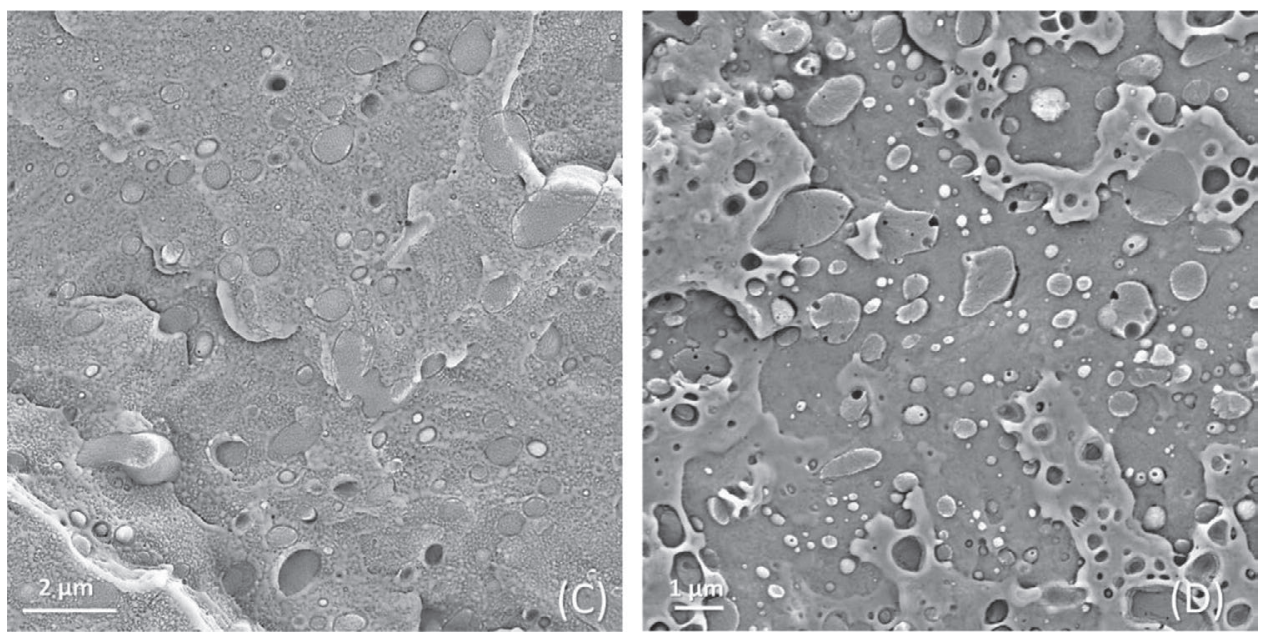

Figure 7. SEM images of Ceramer/Sorbitol system dispersed in PP. Processing conditions $\dot{w}=10 \mathrm{~kg} \mathrm{~h}^{-1}$ for C) and at $\dot{w}=5 \mathrm{~kg} \mathrm{~h} h^{-1}$ for D) with $N=$ 300 rpm. Magnitude: x5000; Power: 5 kV.

by their amount of dispersed phase $(\phi)$ and the processing conditions (flow rate $(\dot{w})$ and screw rotation speed $(N))$. For comparison, these tests have also been performed on the raw PP used in the formulation processed in the same conditions than the other formulations. Young modulus $(E)$, yield stress $\left(\sigma_{\mathrm{y}}\right)$, and strain at break $\left(\varepsilon_{\mathrm{b}}\right)$ obtained are reported in Table 4.

First, it appears that the addition of a dispersed phase seems to alter the mechanical properties. This could be explained by the poor compatibility between the PP phase and the PE-based dispersions. Actually, this kind of blends generally requires a compatibilization between both phases to obtain improved mechanical properties.

Then, by studying the influence of process parameters, it seems that a high flow rate of $10 \mathrm{~kg} \mathrm{~h}^{-1}$ results in a lower
Young's modulus and a higher elongation at break than lower flow rates. Such conditions induce a high shear rate which could induce a degradation of the PP matrix explaining these results. Regarding the screw speed, this parameter seems to have much more influence on the Ceramer 1608/Sorbitol system than on the Ceramer 1608/Jeffamine T-403 system. The main parameter influenced by the rotation speed is the residence time, and although this parameter has an influence on the final reaction speed of the Ceramer 1608/Sorbitol system, it has no influence on that of the Ceramer 1608/ Jeffamine T-403 system because the reaction time is shorter than the residence time and the material therefore stops changing.

Table 4. Mechanical properties of blends processed at different screw rotation speeds $(N)$, different exit flow rates $(\dot{w})$ and for different amounts of dispersed phase $(\phi)$.

\begin{tabular}{|c|c|c|c|c|c|c|}
\hline Material & $\phi[\%]$ & $\dot{w}\left[\mathrm{~kg} \mathrm{~h}^{-1}\right]$ & $N$ [rpm] & $E[\mathrm{MPa}]$ & $\sigma_{\mathrm{y}}[\mathrm{MPa}]$ & $\varepsilon_{\mathrm{b}}[\%]$ \\
\hline \multirow[t]{2}{*}{ Raw extruded PP } & $0 \%$ & 3 & 600 & $1500 \pm 100$ & $34 \pm 1$ & $170 \pm 20$ \\
\hline & $15 \%$ & 3 & 200 & $1300 \pm 100$ & $28 \pm 1$ & $10 \pm 10$ \\
\hline \multirow{11}{*}{$\begin{array}{l}\text { PPH7060 } \\
+ \text { Ceramer } 1608 \\
+ \text { Jeffamine T403 }\end{array}$} & & & 600 & $1300 \pm 100$ & $29 \pm 1$ & $15 \pm 10$ \\
\hline & & & 1000 & $1200 \pm 100$ & $29 \pm 1$ & $20 \pm 10$ \\
\hline & & 5 & 300 & $1400 \pm 100$ & $30 \pm 1$ & $15 \pm 10$ \\
\hline & & & 600 & $1500 \pm 100$ & $30 \pm 1$ & $15 \pm 10$ \\
\hline & & & 1000 & $1400 \pm 100$ & $28 \pm 1$ & $15 \pm 10$ \\
\hline & & 10 & 600 & $1100 \pm 100$ & $29 \pm 1$ & $30 \pm 20$ \\
\hline & & & 1000 & $1000 \pm 200$ & $27 \pm 1$ & $30 \pm 20$ \\
\hline & $30 \%$ & 3 & 200 & $1200 \pm 100$ & $26 \pm 1$ & $10 \pm 10$ \\
\hline & & & 600 & $1100 \pm 100$ & $26 \pm 1$ & $5 \pm 10$ \\
\hline & & 10 & 600 & $1000 \pm 100$ & $24 \pm 1$ & $10 \pm 10$ \\
\hline & & & 1000 & $1000 \pm 100$ & $24 \pm 1$ & $10 \pm 10$ \\
\hline \multirow{4}{*}{$\begin{array}{l}\text { PPH7060 } \\
+ \text { Ceramer } 1608 \\
+ \text { Sorbitol }\end{array}$} & $20 \%$ & 5 & 300 & $1500 \pm 100$ & $32 \pm 1$ & $32 \pm 10$ \\
\hline & & & 800 & $1500 \pm 100$ & $32 \pm 1$ & $10 \pm 10$ \\
\hline & & 10 & 300 & $1400 \pm 100$ & $32 \pm 1$ & $78 \pm 10$ \\
\hline & & & 800 & $1400 \pm 100$ & $32 \pm 1$ & $16 \pm 10$ \\
\hline
\end{tabular}



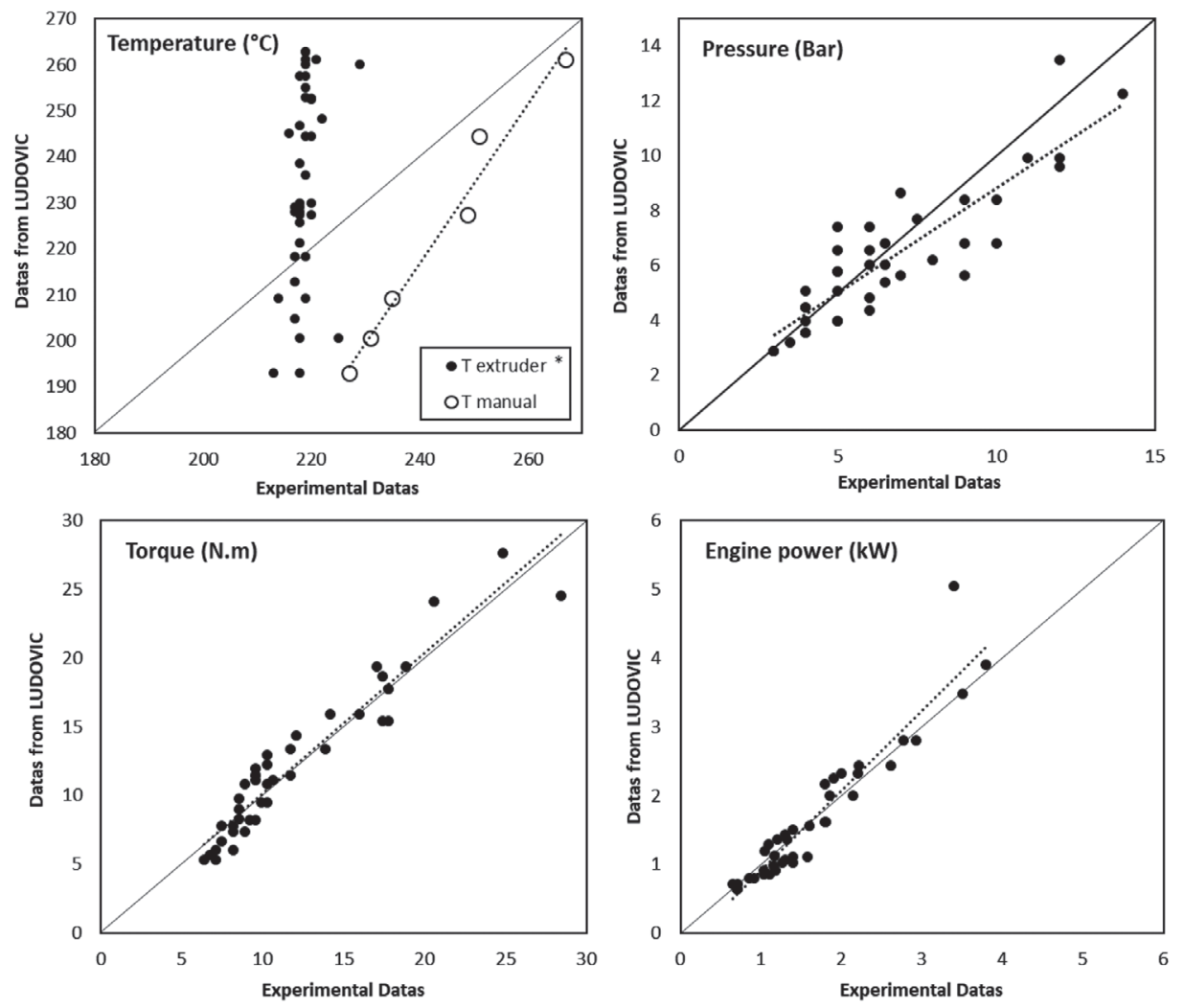

Figure 8. Plots of temperature, engine power, pressure, and torque obtained by the simulation versus these parameters obtained experimentally under different processing conditions. The dotted lines show the correlations between experimental and simulation datas. Reactive system: Ceramer/ Jeffamine phase dispersed in PP. * T extruder: Temperature measured from extruder thermocouple, $\mathrm{T}$ manual: Temperature measured in the bulk polymer at the die exit.

\subsection{Results: LUDOVIC versus Experiments}

Remember that we used the hypothesis that the rheological behavior of the compound material depends only on the properties of the matrix. The simulation has thus been run considering only one single PP phase in the extruder, implying no differences between the different formulations studied. Figure 8 shows, for the system Ceramer/Jeffamine, the comparison between simulation outputs and experimental data. It is worth noting that the 1D global model of Ludovic software model fits well the experiments (around 20\% of maximum error) despite the numerous simplifications and hypothesis implied in this simulation. However, some inaccuracies can be noticed, particularly at high flow rates. The previously mentioned problem of the extruder thermocouple appears clearly in the plot of the temperatures. The temperatures measured manually are more relevant, but they do not perfectly match between the experiments and the model. It can however be explained by the inaccuracy of the experimental data that have been performed with a manual thermocouple at the exit of the extruder, and it is particularly tricky to obtain the exact core temperature of the material this way. Finally, even if the results of the simulation can be enough for most engineering studies, they also can be improved, and machine learning can be a solution to bring this additional accuracy.

Similar comparison between LUDOVIC's results and experimental data for the system Ceramer/Sorbitol is shown in Figure 9. As said earlier, the simulation considers a single PP phase. The same simulation values have thus been used for this comparison. For this system, the simulation seems to fit less the experiments and to over predict the results. Therefore, it appears that the dispersed phase has finally a significant influence on the behavior of the material. Actually, as it can be seen in Figure 3, this reaction is slower and as the viscosities of the reactants are significantly lower than the viscosity of the PP (viscosity ratio of $7.10^{-4}$ between the Ceramer and the PP), we can assume that the reactive system has a lubricant effect on processing condition before creating a dispersed phase of sufficiently high viscosity to be dispersed in the PP matrix. This phenomenon has been described by Cassagnau et al. for various reactive systems. ${ }^{[23]}$ Consequently, we cannot approximate the viscosity of the material as the viscosity of the matrix anymore, but as a lower apparent viscosity (lubricant effect), at least at the early stage of mixing. Finally, it appears that classical modeling simulations are not perfectly adapted to describe polymer blends, especially low viscosity ratio blends.

\subsection{Machine Learning}

In this section, the numerical results associated to the previous section are presented and discussed. The section is structured as follows: the first part addresses the data sets and the 

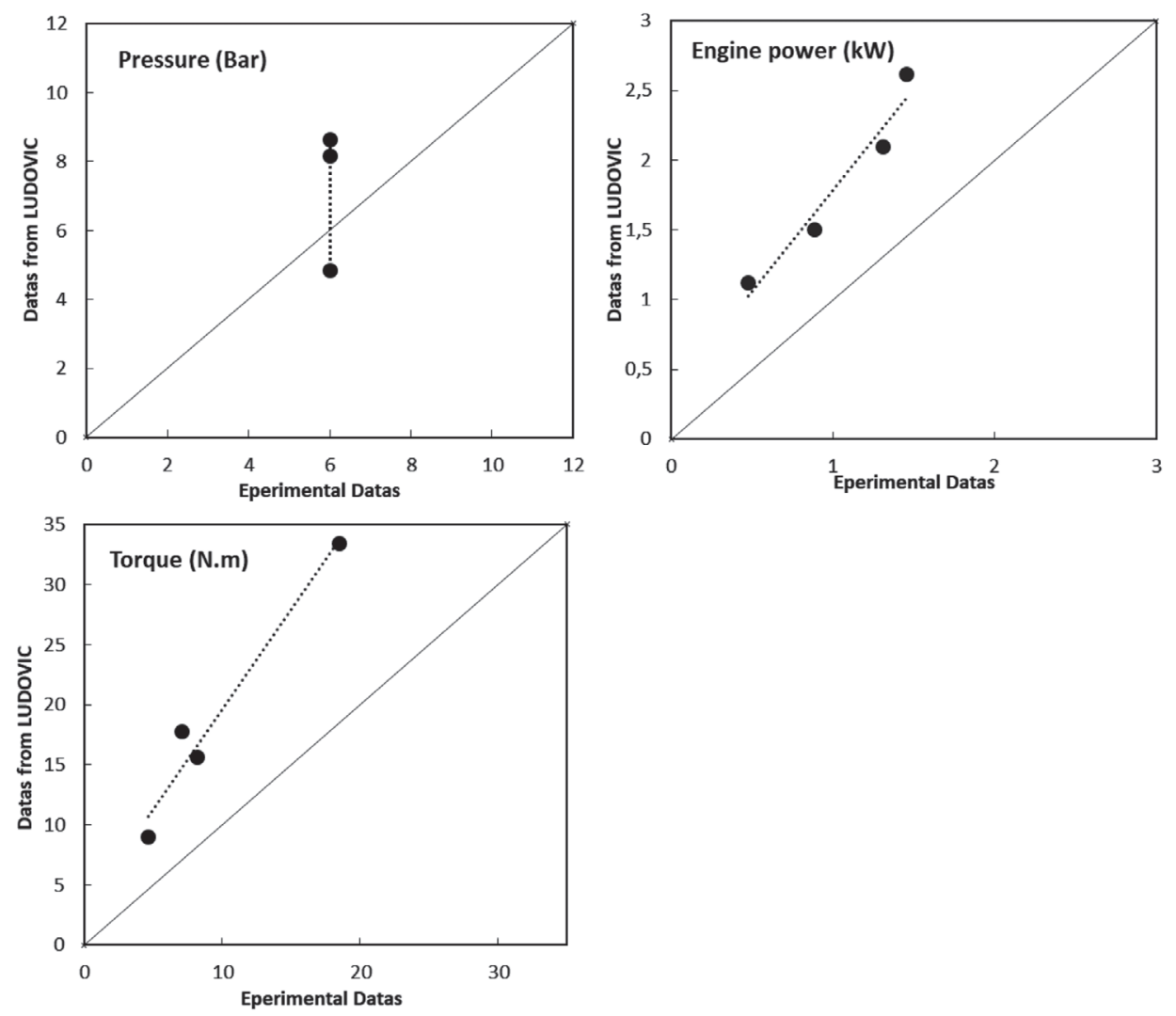

Figure 9. Plots of engine power, pressure, and torque obtained by the simulation versus these parameters obtained experimentally at different flow rates and screw rotation speeds. The dotted lines show the correlations between experimental and simulation datas. Reactive system: Ceramer/Sorbitol system dispersed in PP.

employed numerical algorithms. Then, the results associated with a full data-driven regression and a hybrid-twin solution will be exposed. Finally, some conclusions will be drawn.

\subsubsection{Problem Statement}

Data is coming from two different sources. From one hand, data coming from the Ludovic simulation software, that will be called simulation data. On the other hand, data coming from experimental measures will be referred as experimental data. Both data sets have the same four outputs to be predicted namely, torque, pressure, engine power, and exit temperature. Simulation data has two inputs: flow rate and rotation speed, whereas experimental data, other than the two previous inputs, includes an extra input parameter, the dispersed phase. This last parameter was not considered in the model because of the Ludovic model limitations.

In the following, each of the four outputs will have a ground truth and an approximate model. The basic truth for torque, pressure, and engine power are the experimental measurements, while the approximate model consists of the simulation data. On the contrary, Ludovic software is able to give a more accurate exit temperature measurement since it can extract the temperature at the middle of the die section. Contrarily, the temperature sensor is located on the die wall where the temperature significantly differs from the actual temperature. For that reason, we will consider that the simulation temperature dataset contains the ground truth whereas the experimental measures define the approximate model.

The experimental dataset contains 47 samples (or measurements). The same 47 samples were replicated from simulation (Ludovic). The main goal is to have a regression model which is able to infer the outputs for any point in the parametric space. For that purpose, the initial data set is split into 38 points to construct the regression (training) and 9 points to check the prediction accuracy (test).

As previously reported, we have used two different regression techniques, the sPGD and the Code2Vect - C2V.

Finally, we will compare two different approaches:

- The first, which will be called full data-driven, creates a regression directly on the ground truth.

- The second, which will be called hybrid-twin, involves a regression of the difference between the ground truth and the approximate model. By doing that, a certain amount of the correlations already present in the approximate model were described and therefore, the regression of the deviation is expected to be more linear.

\subsubsection{Full Data-Driven Regression}

As mentioned above, the first approach considers a direct regression on the ground truth without considering the effect of the approximate model. 

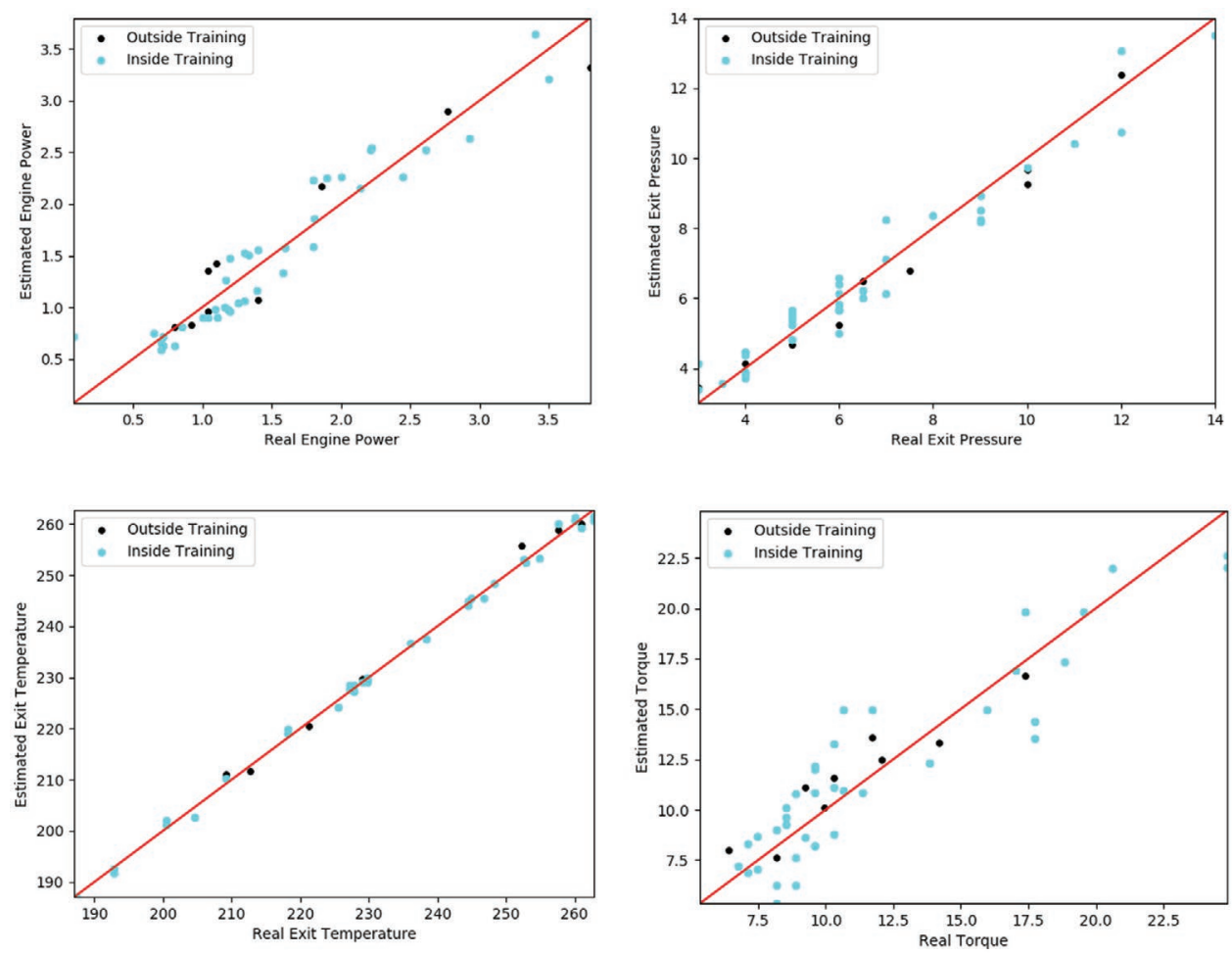

Figure 10. SPGD full data-driven results for engine power (top left), exit pressure (top right), exit temperature (bottom left), and torque (bottom right).

Figure 10 shows the SPGD regression results for the 4 outputs using a full data-driven modeling. The accuracy of the regression is quite acceptable, as the real quantity of interest follows the same tendency than the estimated one. The exit temperature presents less deviation than the other three quantities, due to the fact that the ground truth for the temperature is the simulation
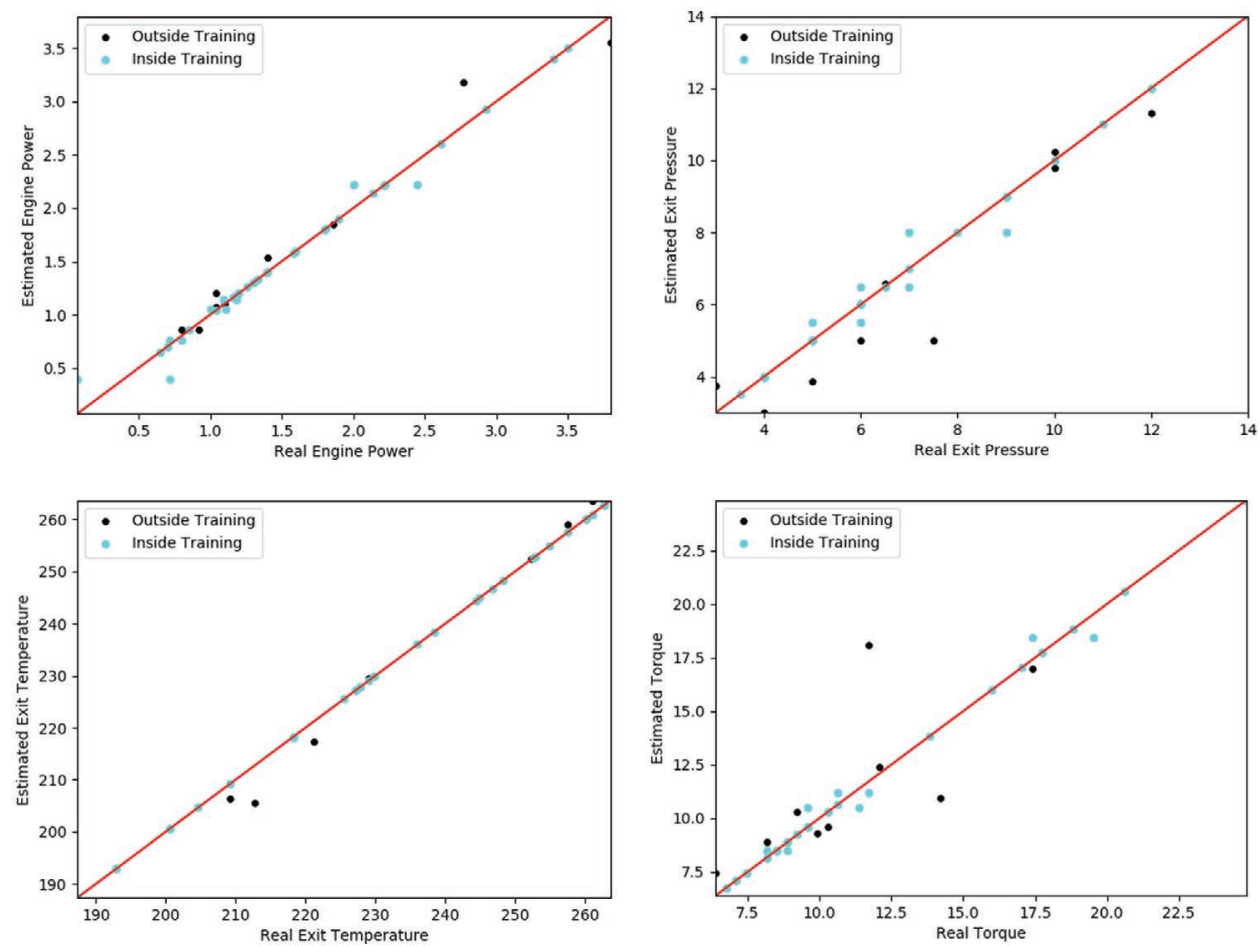

Figure 11. C2V full data-driven results for engine power (top left), exit pressure (top right), exit temperature (bottom left), and torque (bottom right). 
Table 5. Relative error for the full data-driven modeling.

\begin{tabular}{lcccc}
\hline Error DD \% & Engine power & Exit pressure & Torque & Exit temperature \\
\hline SPGD train & 12.6 & 7.2 & 14.0 & 0.4 \\
C2V train & 2.4 & 1.6 & 1.3 & 0.0 \\
SPGD test & 14.9 & 6.5 & 8.8 & 0.6 \\
C2V test & 8.0 & 12.7 & 13.7 & 0.9 \\
\hline
\end{tabular}

dataset, where the use of a deterministic model avoids dispersion. In what follows the computed predictions obtained by the regression for the data used in the regression construction (referred in Figure 9-and the next Figures-as "Inside Training") and those that were not considered in the training (referred in Figure 9 as "Outside Training") are compared with the collected data.

Figure 11 shows the Code2Vect regression results in the full data-driven case. As it can be seen, the results are again acceptable. It can be noticed that $\mathrm{C} 2 \mathrm{~V}$ generates a model that predicts very well in the training points (blue points), however, in some cases the errors in the test dataset (black points) are higher compared with the SPGD results.

Table 5 reports the relative errors for the full data-driven case. The relative error has been normalized with respect to the mean value of each quantity of interest, respectively. As it can be clearly seen, the $\mathrm{C} 2 \mathrm{~V}$ presents less error in the training-set in comparison with the SPGD technique. However, when considering the test-set, the SPGD seems globally more accurate.

\subsubsection{Hybrid-Twin Regression}

The second approach consists of subtracting the approximate model to the ground truth. A regression is applied to that
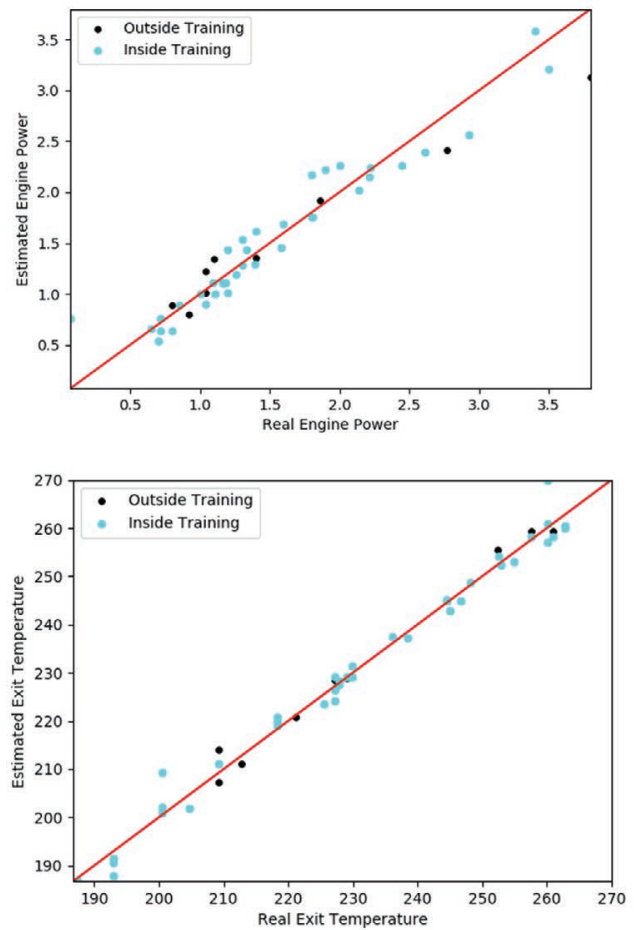

Figure 12. sPGD hybrid-twin results for engine power (top left), exit pressure (top right), exit temperature (bottom left), and torque (bottom right).

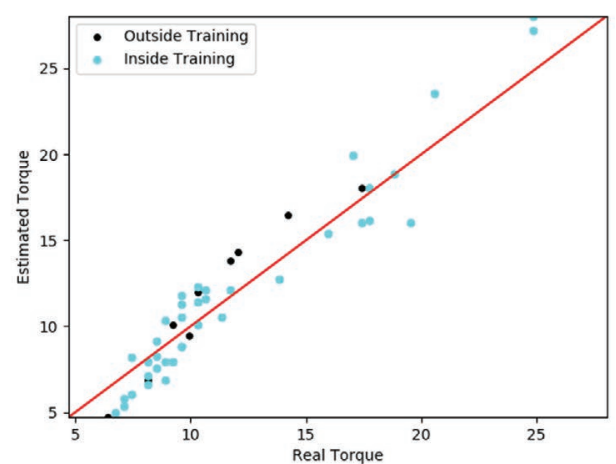

deviation. By doing so, we expect that certain correlations will be already explained by the approximate model facilitating the regression construction and its performances. Thus, the model of the deviation is expected to be easier to capture than the full data-driven route.

Figure 12 shows the estimation of the four outputs when using the hybrid-twin procedure using the sPGD regression to model the deviation. As it can be appreciated, the hybrid-twin methodology behaves slightly better in the training-set. When considering the test-set, the hybrid-twin methodology is on the same level of accuracy than the full data-driven approach.

Figure 13 reports similar results when using the $\mathrm{C} 2 \mathrm{~V}$ for modeling the deviation. As it can be appreciated, C2V model predicts very accurately within the training set. When considering the test set, $\mathrm{C} 2 \mathrm{~V}$ behaves better than sPGD for the engine power and torque.

Table 6 reports a relative error for both C2V and SPGD techniques. As it can be seen, C2V for the torque and sPGD for engine power, perform better than the full data-driven approach. It is also important to highlight that the exit temperature is harder to predict than the full data-driven case. This issue can be explained by the fact that the measured and the computed temperatures do not correspond, one is the bulk material and the other is measured near the die wall.

\section{Conclusion}

The aim of this work was to use machine learning tools combined with a simulation software, based on physical models, to obtain accurate predictions of a complex system with few experimental data. The framework of the present study is the reactive extrusion process for the in situ synthesis of a crosslinked

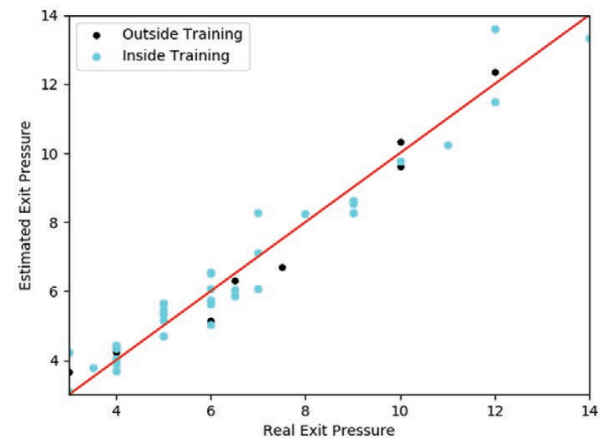



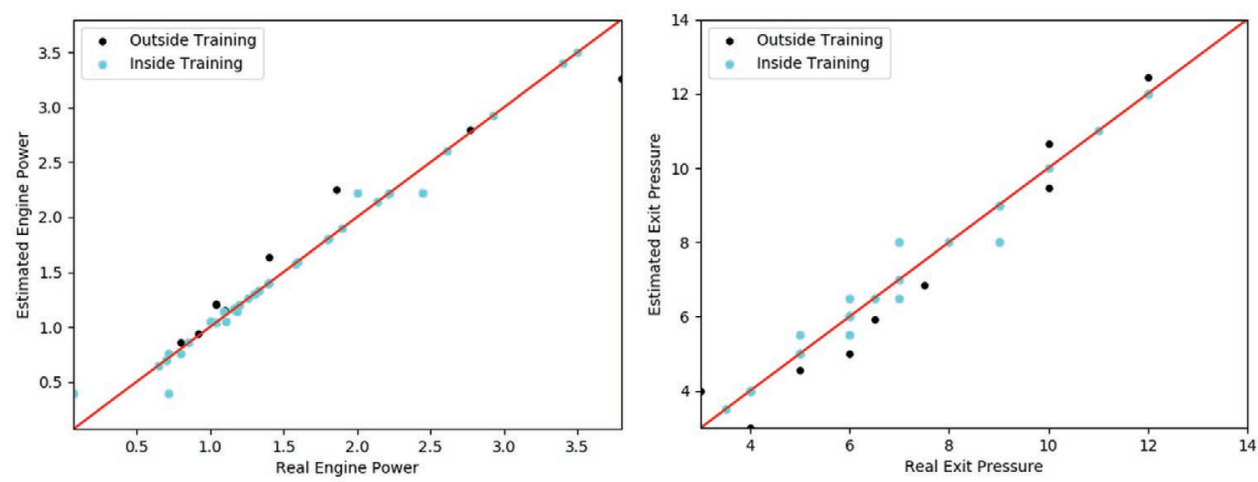

Figure 13. $\mathrm{C} 2 \mathrm{~V}$ hybrid-twin results for engine power (top left), exit pressure (top right), exit temperature (bottom left), and torque (bottom right).

Table 6. Summary of relative error for the hybrid-twin case.

\begin{tabular}{lcccc}
\hline Error HT \% & Engine power & Exit pressure & Torque & Exit temperature \\
\hline SPGD train & 9.8 & 7.4 & 11.3 & 0.9 \\
C2V train & 2.4 & 1.6 & 1.3 & 0.2 \\
SPGD test & 13.0 & 6.8 & 12.6 & 0.8 \\
C2V test & 12.0 & 10.5 & 7.4 & 6.2 \\
\hline
\end{tabular}

dispersed phase. This dispersed phase is based on the reaction between a maleic anhydride functionalized PE oligomer phase with either a triamine or a diol. Viscoelasticity measurements proved that these reactive systems lead to high-density crosslinked networks $\left(\mathrm{Ge} \approx 3 \times 10^{6} \mathrm{~Pa}\right)$. Kinetics studies showed that the reactivity of the first system (Amine based reactions) is really fast $(<10 \mathrm{~s}$ at these processing temperatures) which prevents a good dispersion (coarse morphology) in PP during the extrusion process. The kinetics of the second system (sorbitol-based reaction) being slower, the resulting morphology is finer but a lubricant effect was observed. Finally, in both cases morphologies revealed a lack of affinity between the phases, causing poor properties of the final materials. The twin-screw extrusion 1D software Ludovic was used to model the experiments and it appeared that classical modeling simulations are in some cases not perfectly adapted to describe polymer blends, especially low viscosity ratio blends.

Machine learning methods were then applied to these results in order to improve the predictions. The amount of data being limited (47 samples here), two different regression techniques dealing with low data were experimented: sPGD and Code2Vect (C2V), following two different strategies. The first one, called "full data-driven," creates a regression directly on the ground truth (basically experimental data), and the second one, called "hybrid-twin", involves a regression of the difference between the ground truth and the approximate model (Ludovic software data) that can be seen as a correction of the model. As a result, both strategies and both techniques succeed to bring acceptable predictions of the experiments despite few data. Differences between the two regression techniques are however noticeable depending on the predicted parameter. The hybrid-twin strategy seems also to give slightly better results than the full data-driven one. Moreover, the hybrid-twin strategy has the advantage to predict precisely a complex process whether considering physical phenomenon and theories from the model. It thus combines the logic of physical models with the accuracy of machine learning techniques in low data conditions.

\section{Acknowledgements}

This research was funded by the French ANR through the DataBEST project.

\section{Conflict of Interest}

The authors declare no conflict of interest.

\section{Keywords}

artificial engineering, experimental data, machine learning, polymer processing, reactive extrusion

[1] W. Lertwimolnun, B. Vergnes, Polym. Eng. Sci. 2007, 47, 2100.

[2] P. Cassagnau, V. Bounor-Legaré, F. Fenouillot, Int. Polym. Process. 2007, 22, 218.

[3] J. M. Raquez, R. Narayan, P. Dubois, Macromol. Mater. Eng. 2008, 293, 447.

[4] D. E. Crawford, Beilstein J. Org. Chem. 2017, 13, 65.

[5] P. Cassagnau, V. Bounor-Legaré, B. Vergnes, 2020, 20, 803.

[6] G. Beyer, C. Hopman, Reactive extrusion: Principles and applications, Wiley-VCH Verlag GmbH, Weinheim, Germany 2018.

[7] S. Choulak, F. Couenne, Y. L. Gorrec, C. Jallut, P. Cassagnau, A. Michel, Ind. Eng. Chem. Res. 2004, 43, 7373.

[8] A. Durin, P. D. Micheli, H.-C. Nguyen, C. David, R. Valette, B. Vergnes, Int. Polym. Process. 2014, 29, 641. 
[9] B. Vergnes, G. D. Valle, L. Delamare, Polym. Eng. Sci. 1998, 38, 1781.

[10] B. Vergnes, F. Berzin, C. R. Chim. 2006, 9, 1409.

[11] M. Robert, Ph.D. Thesis, Lyon1 University, 2019.

[12] O. S. Carneiro, J. A. Covas, B. Vergnes, J. Appl. Polym. Sci. 2000, 78 1419.

[13] L. Delamare, B. Vergnes, Polym. Eng. Sci. 1996, 36, 1685.

[14] W. Bahloul, O. Oddes, V. Bounor-Legaré, F. Mélis, P. Cassagnau, B. Vergnes, AlChEJ. 2011, 57, 2174.

[15] G. Della Valle, F. Berzin, B. Vergnes, in Advances in food extrusion technology (Eds: M. Maskan, A. Altan), CRC Press, Boca Raton, Florida, USA 2011, pp. 327-354.

[16] J. Ville, F. Inceoglu, N. Ghamri, J. L. Pradel, A. Durin, R. Valette, B. Vergnes, Int. Polym. Process. 2013, 28, 49.
[17] F. Berzin, B. Vergnes, Front. Mater. 2020, 7, https://doi.org/10.3389/ fmats.2020.00218.

[18] E. Fel, V. Massardier, F. Mélis, B. Vergnes, P. Cassagnau, Int. Polym. Process. 2014, 29, 71.

[19] E. Fel, Ph.D., INSA-Lyon, 2014.

[20] R. Ibáñez, E. Abisset-Chavanne, A. Ammar, D. González, E. Cueto, A. Huerta, J. L. Duval, F. Chinesta, Complexity 2018, 2018, 5608286.

[21] C. A. Martín, R. I. Pinillo, A. Barasinski, F. Chinesta, C. R. Mec. 2019, 347, 754.

[22] F. Chinesta, E. Cueto, E. Abisset-Chavanne, J. L. Duval, F. E.I Khaldi, Arch. Comput. Methods Eng. 2020, 27, 105.

[23] P. Cassagnau, F. Fenouillot, Polymer 2004, 45, 8031. 